Cell Research (1999), 9, 261-269

\title{
NIH 3T3 cells malignantly transformed by mot-2 show inactivation and cytoplasmic sequestration of the p53 protein
}

\author{
Wadhwa ${ }^{1}$ Renu, Syuichi Takano ${ }^{2}$, Youji Mitsui ${ }^{2}$, Sunil \\ C KAUL ${ }^{2}$ \\ 1. Chugai Research Institute for Molecular Medicine, 153-2 \\ Nagai, Niihari-Mura, Ibaraki 300-41, Japan \\ 2. National Institute of Bioscience and Human-Techno logy, \\ AIST, 1-1 Higashi, Tsukuba, Ibaraki 305-8566, Japan
}

\begin{abstract}
In previous studies we have reported that a high level of expression of mot- 2 protein results in malignant transformation of NIH 3T3 cells as analyzed by anchorage independent growth and nude mice assays [Kaul et al., Oncogene, 17, 90711, 1998]. Mot-2 was found to interact with tumor suppressor protein $\mathrm{p} 53$. The transient overexpression of mot-2 was inhibitory to transcriptional activation function of p53 [Wadhwa et al., J. Biol. Chem., 273, 29586-91, 1998]. We demonstrate here that mot-2 transfected stable clone of NIH 3T3 that showed malignant properties indeed show inactivation of $\mathrm{p} 53$ function as assayed by exogenous p53 dependent reporter. The expression level of p53 in response to UV-irradiation was lower in NIH 3T3/mot-2 as compared to NIH 3T3 cells and also exhibited delay in reaching peak. Furthermore, upon serum starvation p53 was seen to translocate to the nucleus in NIH 3T3, but not in its mot-2 derivative. The data suggests that mot-2 mediated cytoplasmic sequestration and inactivation of p53 may operate, at least in part, for malignant phenotype of NIH 3T3/ mot- 2 cells.
\end{abstract}

\footnotetext{
* Corresponding author: Renu Wadhwa, Chugai Research Institute for Molecular Medicine, 153-2 Nagai, Niihari-Mure,Ibaraki 300-41, Japan Tel: +81 29830 6211; Fax +81 29830 6270. E-mail: renu@cimmed.com
} 
NIH 3T3/mot-2 inactivation of the p53 protein

Key words: NIH 3T3, mot-2, p53 inactivation, malignant transformation.

\section{INTRODUCTION}

Involvement and inactivation of p53, a tumor suppressor and cellular transcription factor in cellular immortalization has been well documented[1-4]. Various activities of p53 protein involve its functional domains, viz., an amino- terminus transactivation domain (amino acids [a. a.] 1-44), a sequence specific DNA binding domain (a. a. 100-300), a carboxy-terminus oligomerization domain and a regulatory domain (a. a. 319-393). Wild type p53 binds to DNA and interacts with components of basal transcription factor TFIID to activate transcription of its down stream genes such as, $\mathrm{p} 21^{\mathrm{WAF}-1}, \mathrm{mdm} 2$ and GADD45. Most of the mutant p53 proteins lack the ability to interact with TATA boxbinding protein-associated factors TAFII32 and TAFII70 although in many cases these can still bind to DNA. Besides, conformation of p53 and its interactions with other proteins modulate its various functions[5],[6]. Several cellular proteins including some of the hsp70 family members have been shown to interact with p53[7],[8-13]. Although mutational or mdm-2 mediated inactivation of p53 is a common event involved in cellular transformation[1], p53 is inactivated in a considerable number of tumors/transformed cells by an unknown mechanism(s).

We initially cloned mortalins, mot-1 and mot-2, coding for pancytosolically and perinuclearly distributed members of the hsp70 family of proteins, from normal and immortal murine cells, respectively[14],[15]. The open reading frames of the two types of murine mortalins differ in two nucleotides, encode proteins differing in two amino acids, and have contrasting biological activities[16]. RNA in situ hybridization and immunohistochemical studies on mortalin in normal murine tissues showed a higher level of expression in nondividing cell populations than in dividing cells. However, tumor tissues were seen to have a high intensity of mortalin staining by an antibody that reacts with both mot-1 and mot-2 proteins[17],[18]. Mortalin was also identified as PBP-74, mtHSP70 and Grp75, and has been assigned roles in antigen processing, in vivo nephrotoxicity and radioresistance in independent studies from other groups[19],[20]. We have earlier demonstrated functional interactions of mot-2 protein with wild type p53[7]. Colocalization of wild type p53 and mortalin protein was observed in transformed (nonpancytosolic mortalin) but not in normal (pancytosolic) cell types. The transcriptional activation function of p53 was impaired by transfections of mot-2, but not mot-1, protein suggesting a novel mechanism of p53 inactivation by mot-2 protein. In the present study, we demonstrate that mot-2 transformed NIH 3T3 cells[21] show inactivation and cytoplasmic sequestration of the p53 protein. 
Wadhwa $\mathrm{R}$ et al.

\section{MATERIAL AND METHODS}

\section{Cell culture and UV-irradiation}

Normal mouse embryonic fibroblasts from the CD1-ICR strain mouse (CMEF), and immortal clone (RS-4) established from CMEF, NIH 3 T3 cells, and embryonic fibroblasts from a p53-null (p53-/-) mouse were cultured in Dulbecco' s modified Eagle' s minimal essential medium supplemented with $10 \%$ fetal calf serum (Gibco), at $37^{\circ} \mathrm{C}$ in a humidified atmosphere of $95 \%$ air and $5 \% \mathrm{CO}_{2}$. NIH 3T3/mot-2 transfectants were selected in DMEM supplemented with $10 \%$ FCS and $500 \mathrm{mg} / \mathrm{ml} \mathrm{G} 418$. For UV-irradiation, cells grown to about $50 \%$ confluency in $100-\mathrm{mm}$ dishes were rinsed once with phosphate-buffered saline (PBS) and irradiated $\left(10 \mathrm{~mJ} / \mathrm{cm}^{2}\right)$ by placing the dish in UV cross-linker (Funakoshi, Japan). The original medium was replaced and the cells were returned to $37^{\circ} \mathrm{C}$. At indicated post-irradiation time points, cells were harvested in Nonidet P-40 lysis buffer (20 mM Tris (pH 7.5), 1 mM EDTA, 1 mM EGTA, $0.1 \mathrm{mM}$ phenylmethylsulfonylfluoride, $150 \mathrm{mM} \mathrm{NaCl}, 1 \%$ Nonidet $\mathrm{P}-40$ ) for $30 \mathrm{~min}$ on ice.

\section{Western blot analysis}

The protein sample $(20-40 \mu \mathrm{g})$ separated on SDS-polyacrylamide gel was electroblotted onto nitrocellulose membrane using a semidry transfer blotter. Immunoassays were performed with antimortalin[14], anti-p53 (PAb-421; Calbiochem) and anti-actin (Boehringer Mannheim) antibodies.

\section{Reporter assays}

Cells grown in 12 -well plates were transfected with $0.5 \mu \mathrm{g}$ of p53-responsive, pWWP-luc or PG13-luc (kindly provided by Dr. Bert Vogelstein)[22] or control (pGVB2; Promega) reporter plasmid. $48 \mathrm{~h}$ after transfection, luciferase assays (Dual-Luciferase ${ }^{T M}$ Reporter Assay System, Promega) were performed. Luciferase values were calculated per microgram of the protein as determined by Bradford protein assay. p53 responsive CAT reporter plasmid pMCKCAT and a control plasmid, pCAT ${ }^{\mathrm{R}}$ (Promega) were also transfected to NIH $3 \mathrm{~T} 3$ and its mot-2 derivative cells. Reporter activity was measured by chloramphenicol acetyltransferase (CAT) ELISA (Boehringer Mannheim). To examine the internal transfection efficiency of cells, cotransfections of $\mathrm{pRL}$ CMV (Promega) or pCMV.SPORT- $\beta$-gal plasmid (Gibco) were employed in both the experiments. $\beta$-gal activity was monitored by $\beta$-gal enzyme assay (Promega). Error bars represent standard deviation $[n=4]$.

\section{Immunofluorescence and fluorescence digital imaging microscopy}

Cells were double-stained with monoclonal anti-p53 (PAb421; Calbiochem) and polyclonal antimortalin antibodies, and visualized by secondary staining with fluorescein isothiocyanate (FITC)conjugated sheep anti-mouse IgG and Texas Red (TRITC)-conjugated donkey anti-rabbit IgG (Amersham). Three-dimensional images with enhanced fluorescence were obtained using laser digital imaging microscopy with a $40 \mathrm{X}$ Plan-NEOFLUAR objective on a Zeiss Axiophot microscope (Carl Zeiss, Germany) equipped with a CELLscan System (Scanalytics, Billerica, MA)[23]. The extent to which the two proteins overlapped was assessed by combining the two images using computer graphics software. The individual mortalin and p53 images were seen as red and green fluorescence, respectively, and the colocalized proteins appeared yellow under this program.

\section{RESULTS AND DISCUSSION}

NIH 3T3 and their malignantly transformed stable derivative clone, NIH 3T3/mot-2 were assayed for mot-2 and p53 proteins by Western blotting. As expected mot-2 was 
NIH 3T3/mot-2 inactivation of the p53 protein

expressed at a high level in NIH 3T3/mot-2 cells. p53 protein remained undetected in NIH 3T3/mot-2 cells by Western blotting implying that it is not mutated during the course of malignant transformation[21],[24]. NIH 3T3, its mot-2 derivative, normal mouse embryonic primary fibroblasts (CMEF) and its immortal mouse cell line, RS-4 that is shown to have mutant p53, were subjected to p53-responsive luciferase reporter assay along with p53-/- mouse embryonic fibroblasts. As expected, CMEF and NIH 3T3 cells showed wild type p53 responsive reporter activity and no p53 activity was detected in RS-4 and p53-/ - cells (Fig 1A). NIH 3 T3/mot-2 cells revealed inactivation of p53 function. In four independent experiments in which the differences in transfection efficiency of NIH 3T3 and NIH 3T3/mot-2 were normalized by cotransfection of pCMVbgal reporter plasmid, a significant repression (about ten-fold) of p53 transactivation function was detected in NIH 3T3/mot-2 (Fig 1B). A control p53-independent reporter plasmid, pGVB2, showed two-fold lower activity in NIH 3T3/mot-2 cells. Reporter assays were also performed using a synthetic p53 responsive CAT reporter plasmid, pMCKCAT. Similar results, i. e., 10 -fold repression of p53 responsive transactivation in NIH 3T3/mot-2 cells were obtained in three independent experiments (Fig 1C). A control p53-independent reporter plasmid, pCAT, with a high level of basal activity showed two-fold lower activity in NIH $3 \mathrm{~T} 3 /$ mot- 2 cells. The data demonstrated that mot- 2 overexpression in NIH 3 T3 cells re-

A

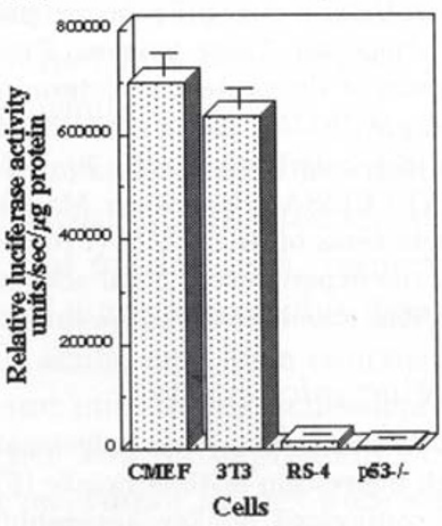

B

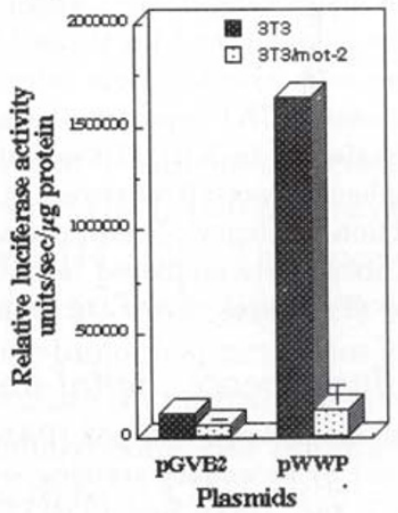

$\mathrm{C}$

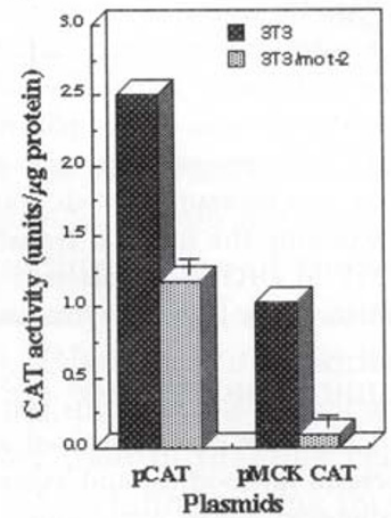

Fig 1.

NIH 3T3, RS-4, CMEF and p53-/- cells were plated in 12-well dishes, for $24 \mathrm{~h}$ and then transfections were performed using Lipofectamine ${ }^{T M}$ (Gibco). $0.5 \mu \mathrm{g}$ of p53-responsive reporter plasmid containing 13 repeats of wild type p53 binding consensus sequence, PG13-luc (a kind gift from Dr. Bert Vogelstein) and pRL-CMV (Promega), an internal control to determine the efficiency of transfections were used. $48 \mathrm{~h}$ after transfection, luciferase assays (Dual-Luciferase ${ }^{T M}$ Reporter Assay System, Promega) were performed. Luciferase values were calculated per microgram of the protein as determined by Bradford protein assay. All reporter assays were performed four times. 
Wadhwa $\mathrm{R}$ et al.

sulted in inhibition of p53-induced transactivation. Such abrogation of some or all of normal tumor suppressor activity of p53 may be responsible, at least in part, for their malignant phenotype of NIH3T3/mot-2 cells. Recently, inactivation of p53 by HHV-6 has been reported to result in malignant transformation of NIH 3T3 cells[25]. Furthermore, RS-4 cells that have a higher expression level of mot-2 than NIH 3T3 cells and possess mutant p53 do not show malignant phenotype (unpublished observations) suggesting that the transforming activity of mot-2 may be mediated mostly by inactivation of wild type p53.

Wild type p53 is rapidly induced in response to UV-irradiation in a dose dependent manner. This has been frequently used as a measure for transcriptional activation function of p53. To investigate p53 activity in NIH 3T3 cells and its mot-2 stable derivative, we next assayed their response to UV-irradiation. Expression of p53 was seen to increase upon UV-irradiation in both the cell types. This was consistent with the wild type p53 status of these cells as determined by Western blotting and p53-dependent reporter assays described above. Both cell types showed initial increase in p53 at the same time point implying that they are comparable in their response to UV-irradiation. However, the time required to reach peak level of p53 expression following UV-irradiation was different in two cell types. Whereas in NIH 3T3 cells a peak level of p53 expression was

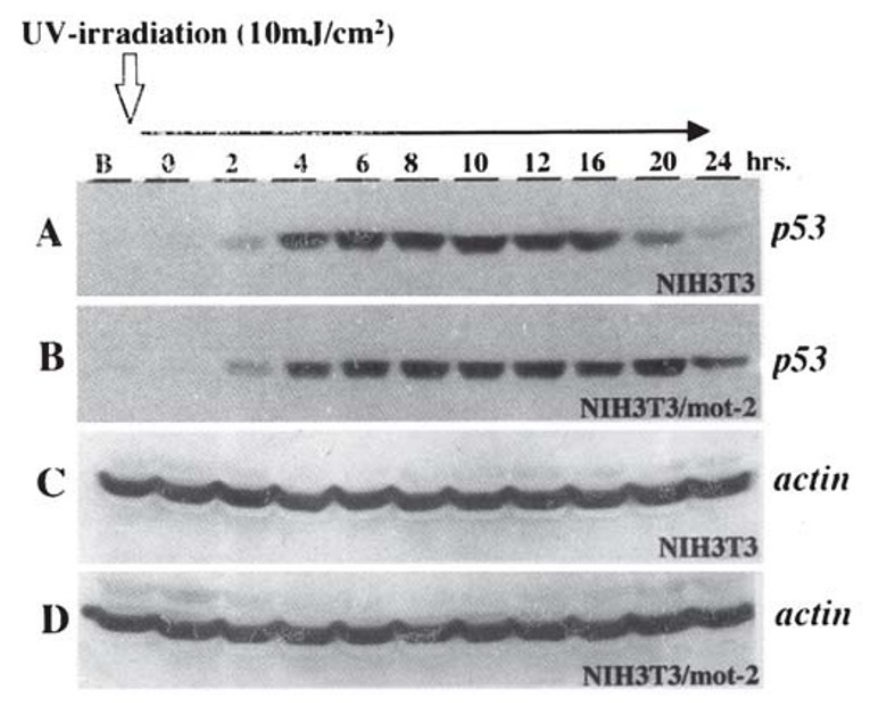

Fig 2.

Western blotting of cells exposed to UV-irradiation $\left(10 \mathrm{~mJ} / \mathrm{cm}^{2}\right)$. Mot-2 was seen to increase gradually in NIH $3 \mathrm{~T} 3$ as well as its mot-2 derivative. At corresponding time intervals ranging 4-16 h, p53 levels were low in NIH 3T3 /mot2 cells. Actin was used as a loading control. 
NIH 3T3/mot-2 inactivation of the p53 protein

detected at 6-10 h, NIH 3T3/mot-2 cells exhibited a somewhat delayed response with a peak detected at about $20 \mathrm{~h}$ (Fig 2). These data imply that whereas in NIH 3T3 cells the peak of UV-irradiation induced growth arrest of cells will occur at $10 \mathrm{~h}$, its mot-2 derivative will be maximally arrested at $20 \mathrm{~h}$ post-irradiation. At early time points ranging 2-10 h post UV-irradiation, NIH 3T3/mot-2 cells showed comparably lower steady state level of p53 and somewhat slower increase in p53 as compared to NIH 3T3 cells. These results provide an evidence that mot-2 may act by reducing p53 steady-state levels. This was in agreement with a lower level of p53 expression in COS7 cells transiently tranfected with mot-2 expression plasmid[7]. It is suggestive that a high level of mot-2 expression may target some of p53 for degradation by inhibiting its nuclear translocation as shown below and therefore may limit response of cells to genotoxic insults.

Double immunofluorescence studies for mot-2 and p53 revealed a high intensity of perinuclear mortalin in NIH 3T3/mot-2 as compared to NIH 3T3 cells (Fig 3A). In an unsynchronized culture of NIH 3T3 cells approximately 30-40\% of cells were seen to have nuclear staining of p53, the cytoplasmic staining was barely visible under epifluorescence microscope. In case of mot-2 stable transfectants, only 6-10\% of cells were seen to have nuclear staining (Fig 3A). Next, G1 arrested cells (confirmed by FACS analysis) obtained by culture in DMEM-supplemented with $0.1 \%$ FBS for $48 \mathrm{~h}$, were fixed for immunostaining with anti-p53 and anti-mortalin antibodies. More than $80 \%$ of the serum-starved control NIH 3T3 cells were seen to have nuclear localization of p53 whereas less than $20 \%$ of mot-2 overexpressing cells showed nuclear translocation of p53 under these conditions (Fig 3B). The data suggested cytoplasmic retention/abrogation of nuclear translocation of p53 in mot-2 overexpressing NIH 3T3 cells. Taken together with reporter assays these results have suggested the mot-2 mediated abrogation of nuclear translocation and inactivation of p53 function. Functional inactivation of wild-type p53 by its abnormal cytoplasmic sequestration has been reported in a subset of neuroblastomas[26], the mechanism of this sequestration has not been elucidated. Ostermeyer et al[27] have reported that the $\mathrm{C}$-terminus of p53 is involved in cytoplasmic aggregates in neuroblastomas. We have observed coimmunolocalization of p53 and mortalin in the cytoplasm of human glioblastoma cells, SY-5Y, A-172 and YKG-1[2].

\footnotetext{
$\triangleright$ Fig 3.

A, Cells were double stained with monoclonal anti-p53 (PAb421; Calbiochem) and polyclonal antimortalin antibodies, and visualized by secondary staining with FITC-conjugated sheep anti-mouse IgG and TRITC-conjugated donkey anti-rabbit IgG (Amersham Corp.). Immunofluorescence as observed under epifluorescence microscope is shown.

$\mathrm{B}$, Three-dimensional images with enhanced fluorescence were obtained using laser digital imaging microscopy with a $40 \mathrm{X}$ Plan-NEOFLUAR objective on a Zeiss Axiophot microscope (Carl Zeiss, Germany) equipped with a CELLscan System (Scanalytics, Billerica, MA). The extent to which the two proteins were similarly distributed was assessed by combining the two images using computer graphics software. The individual mortalin and p53 images were seen as typical red and green fluorescence and the colocalized regions appeared yellow under this program.
} 
Wadhwa $\mathrm{R}$ et al.

A
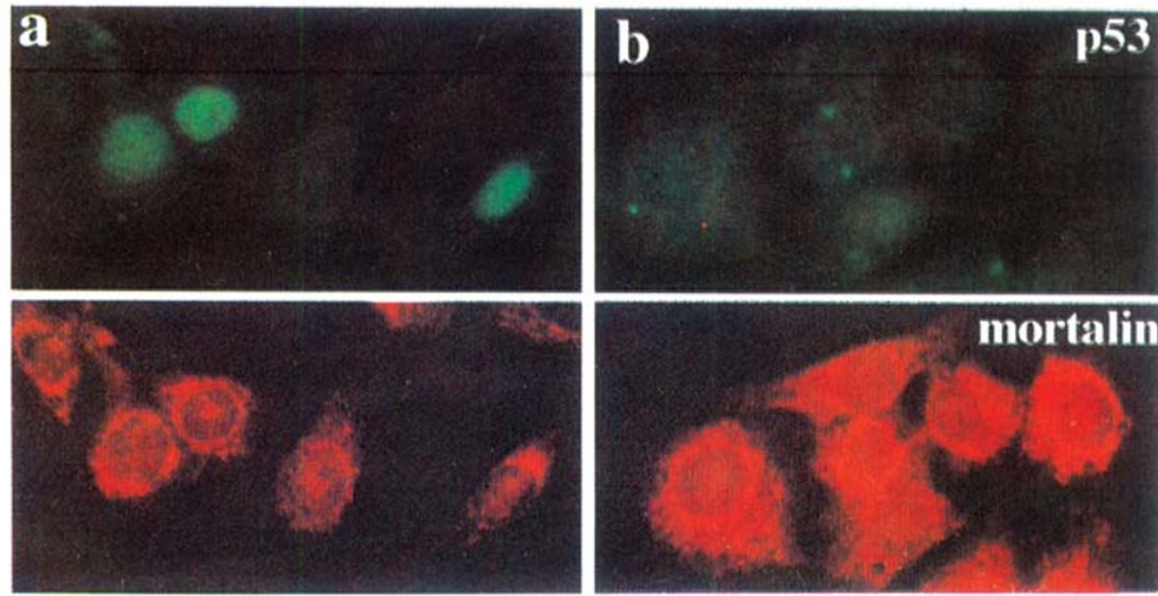

NIH 3T3

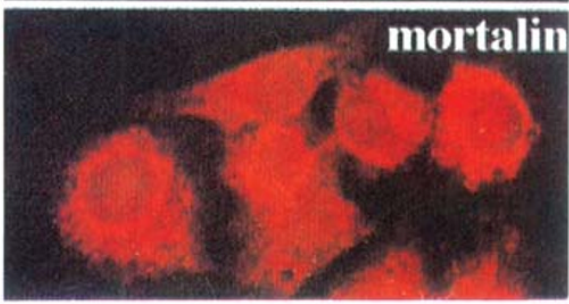

NIH $3 \mathrm{~T} 3 / \mathrm{m} 2$

B
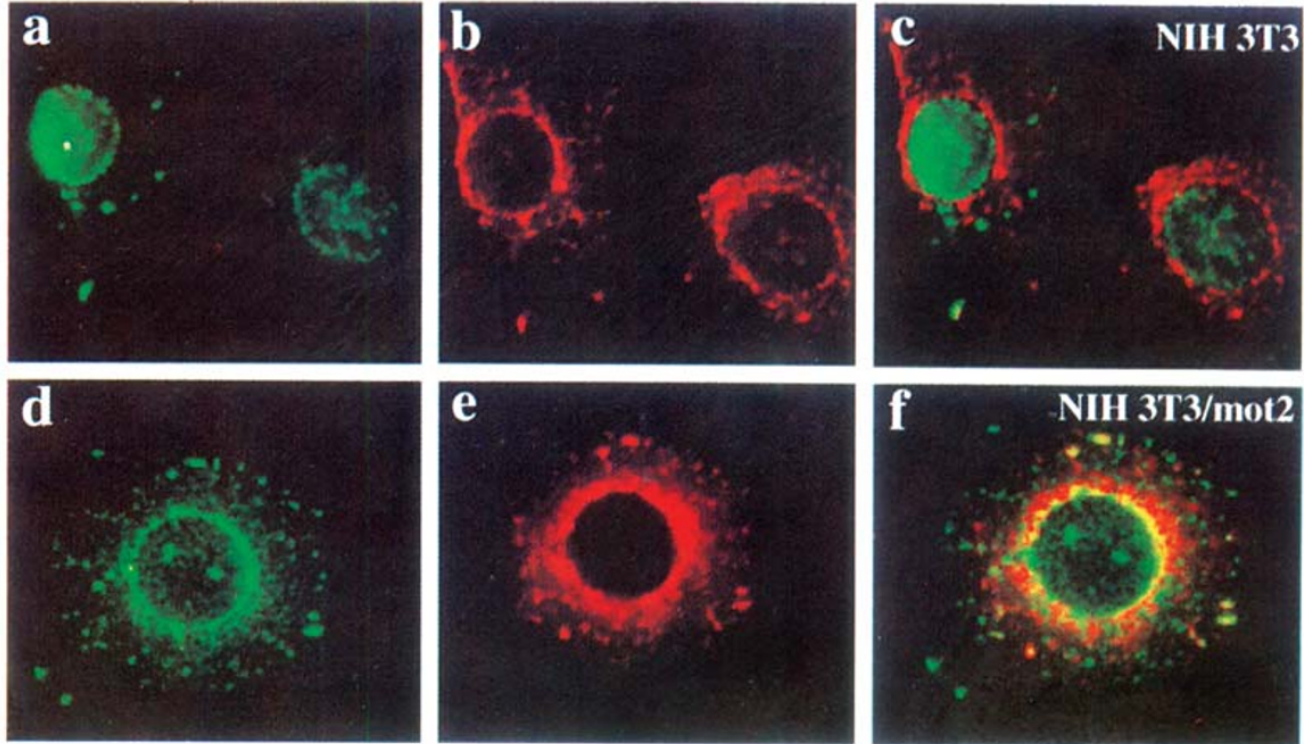

p53

e

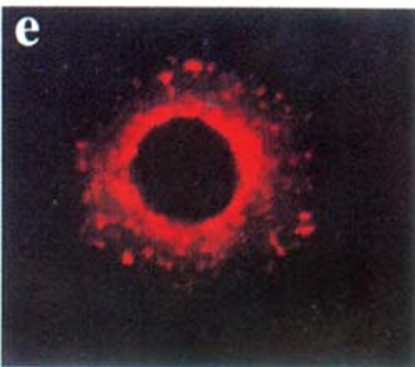

mortalin

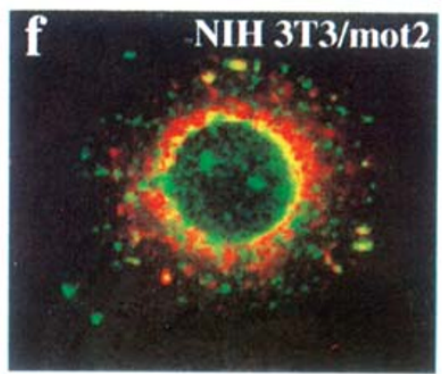

overlay

Percent cells showing nuclear p53: NIH 3T3 $\sim 80 \%$ NIH 3T3/mot-2 $20 \%$ 
NIH 3T3/mot-2 inactivation of the p53 protein

Interestingly, p53 lacking in its C-terminus did not bind to mot-2 in in vitro mot-2/p53 binding assays (unpublished observations). Taken together, it is suggestive that mortalin may be involved in abnormal cytoplasmic sequestration and inactivation of wild type p53 in these cells.

In summary, we have shown nuclear exclusion and reduced steady-state level of p53 in NIH 3T3 cells malignantly transformed by stable expression of mot-2. Such mot-2 mediated inactivation of wild type p53 may operate, at least in part, for their malignant properties.

\section{ACKNOWLEDGMENTS}

We thank Akiko Yoshida and Tomoko Yaguchi for excellent technical assistance.

\section{REFERENCES}

[1] Levine AJ. p53, the cellular gatekeeper for growth and division. Cell 1997; 88:323-31.

[2] Harvey DM, Levine AJ. p53 alteration is a common event in the spontaneous immortalization of primary BALB/c murine embryo fibroblasts. Genes Dev 1991; 5:2375-85.

[3] Metz T, Harris AW, Adams JM. Absence of p53 allows direct immortalization of hematopoietic cells by the myc and raf oncogenes. Cell 1995; 82:29-36.

[4] ynford-Thomas D. Telomeres, p53 and cellular senescence, Oncology Res. 1996; 8:387-98.

[5] Donehower LA, Bradley A. The tumor suppressor p53. Biochim Biophy Acta 1993; 23:181-205.

[6] Adler V, Pincus MR, Minamoto T, Fuchs SY, Bluth MJ, Brandt -Rauf PW, Friedman FK, Robinson RC, Chen JM, Wang XW, Harris CC, Ronai Z. Conformation-dependent phosphorylation of p53. Proc Natl Acad Science USA 1997; 94:1686-91.

[7] Wadhwa R, Takano S, Robert M, Yoshida A, Reddel RR, Nomura H, Mitsui Y, Kaul SC. Inactivation of tumor suppressor p53 by mot-2, an hsp70 family member. J Biol Chem 1998; 273:29586-91.

[8] Hinds PW, Finlay CA, Frey AB, Levine AJ. Immunological evidence for the association of p53 with a heat shock protein, hsc70, in p53-plus-ras-transformed cell lines. Mol Cell Biol 1987; 7:2863-9.

[9] Kaur J, Srivastava A, Ralhan R. p53-HSP70 complexes in oral dysplasia and cancer: potential prognostic implications. Europ J Cancer B Oral Oncol 1996; 32:45-9.

[10] Sugito K, Yamane M, Hattori H, Hayashi Y, Tohnai I, Ueda M, Tsuchida N. and Ohtsuka K. Interaction between hsp70 and hsp40, eukaryotic homologues of DnaK and DnaJ, in human cells expressing mutant-type p53. FEBS Lett 1995; 358:161-4.

[11] Hainaut P, Milner J. Interaction of heat- shock protein 70 with p53 translated in vitro: evidence for interaction with dimeric p53 and for a role in the regulation of p53 conformation. EMBO J 1992; 11: 3513-20.

[12] Fourie AM, Hupp TR, Lane DP, Sang BC, Barbosa MS, Sambrook JF, Gething MJ. HSP70 binding sites in the tumor suppressor protein p53. J Biol Chem 1997; 272:19471-9.

[13] Merrick BA, He C, Witcher LL, Patterson RM, Reid JJ, Pence -Pawlowski PM, Selkirk JK. HSP binding and mitochondrial localization of p53 protein in human HT1080 and mouse C3H10T1/2 cell lines. Biochim Biophys Acta 1996; 13:57-68.

[14] Wadhwa R, Kaul SC, Ikawa Y, Sugimoto Y. Identification of a novel member of mouse hsp70 family. Its association with cellular mortal phenotype. J Biol Chem 1993; 268:6615-21.

[15] Wadhwa R, Kaul SC, Mitsui Y, Sugimoto Y. Differential subcellular distribution of mortalin in mortal and immortal mouse and human fibroblasts. Exp Cell Res 1993; 207:442-8.

[16] Wadhwa R, Kaul SC, Sugimoto Y, Mitsui Y. Induction of cellular senescence by transfection of cytosolic mortalin cDNA in NIH 3T3 cells. J Biol Chem 1993; 268:22239-42. 
Wadhwa $\mathrm{R}$ et al.

[17] Kaul SC, Matsui M, Takano S, Sugihara T, Mitsui Y, Wadhwa R. Expression analysis of mortalin, a unique member of the Hsp70 family of proteins, in rat tissues. Exp Cell Res 1997; 232:56-63.

[18] Takano S, Wadhwa R, Yoshii Y, Nose T, Kaul SC, Mitsui Y. Elevated levels of mortalin expression in human brain tumors. Exp Cell Res 1997; 237:38-45.

[19] Kaul SC, Mitsui Y, Wadhwa R. Molecular insights to cellular mortality and immortalization. Ind J Exp Biol 1998; 36:345-52.

[20] Wadhwa R, Kaul SC, Mitsui Y. (1999) Cellular mortality and immortalization: a complex interplay of multiple gene functions. In: Progress in Molecular and Subcellular Biology, Vol. 24, Cell Immortalization (Edited by Dr. A. Macieira-Coelho) Springer Verlag, Germany. 191-204.

[21] Kaul SC, Duncan EL, Englezou A, Takano S, Reddel RR, Mitsui Y, Wadhwa R. Malignant transformation of NIH3T3 cells by overexpression of mot-2 protein. Oncogene 1998; 17:907-11.

[22] El-Deiry WS, Tokino T, Velculescu VE, Levy DB, Parsons R, Trent JM, Lin D, Mercer WE, Kinzler KW, Vogelstein, B. WAF1, a potential mediator of p53 tumor suppression. Cell 1993; 75:817-25.

[23] Fay FS, Carrington W, Iogarty KE. Three-dimensional molecular distribution in single cells analyzed using the digital imaging microscope. J Microsc 1989; 153:133-49.

[24] Kaul SC, Wadhwa R, Sugihara T, Obuchi K, Komatsu Y, Mitsui Y. Identification of genetic events involved in early steps of immortalization of mouse fibroblasts. Biochim Biophys Acta 1994; 1201:38996.

[25] Kashanchi F, Araujo J, Doniger J, Muralidhar S, Hoch R, Khleif S, Mendelson E, Thompson J, Azumi N, Brady JN, Luppi M, Torelli G, Rosenthal J. Human herpesvirus 6 [HHV-6] ORF-1 transactivating gene exhibits malignant transforming activity and its protein binds to p53. Oncogene 1997; 14:35967.

[26] Moll UM, Ostermeyer AG, Haladay R, Winkfield B, Frazier M, Zambetti, G. Cytoplasmic sequestration of wild-type p53 protein impairs the G1 checkpoint after DNA damage. Mol Cell Biol 1996; 16:112637.

[27] Ostermeyer AG, Runko E, Winkfield B, Ahn B, Moll UM. Cytoplasmically sequestered wild-type p53 protein in neuroblastoma is relocated to the nucleus by a C-terminal peptide Proc. Natl Acad Sci USA 1996; 93:15190-94.

Received Oct-30-1999.

Accepted Nov-5-1999. 